\title{
Catch twin nucleation in action at atomic scale
}

\author{
Yuntian Zhu
}

Understanding the deformation mechanisms of nanocrystalline (nc) metals at the atomic-scale is crucial for determining how far the strengths of metals can be ultimately reached. Although several breakthroughs have been achieved by designing nc metals with ultra-high strength and high ductility [1], the atomistic deformation mechanisms of nc metals with grain sizes of $<15 \mathrm{~nm}$ have not been fully understood because of the lack of direct atomic-scale experimental evidence [1,2]. For a long time, our understanding of the atomistic deformation mechanisms of nc metals relied heavily on molecular dynamic simulations, which had been the only method for revealing atomic-scale dynamic plastic deformation processes of nc materials [2] before Han and his colleagues developed an experimental technique that allows direct atomic resolution imaging of dynamic tensile deformation processes of nc materials $[3,4]$. Using this technique, they discovered that the critical size for deformation mode transformation from intra-grain dislocation activities to inter-grain plasticity can be as small as $\sim 6 \mathrm{~nm}$, which is far below $\sim 15$ nmpredicted by MD simulations $[1,2]$. The discovery set up a milestone for the strength limit of polycrystalline materials since the inverse HallPetch deformation mode was proposed about three decades ago, which indicated the strength of polycrystalline materials could continue to increase by decreasing grain size down to $\sim 6 \mathrm{~nm}$. This provides the direct atomic-scale experimental evidence for the upper limit strength of nc metals and important guidance for developing bulk structural materials with ultra-high strength yet with high ductility.

With the capability of atomic-scale in situ investigation of the deformation mechanisms of nc metals, a long-time puzzle in nc metals, the deformation twinning nucleation process in high stacking fault energy metals is revealed unambiguously [5]. It has been believed that a deformation twin nucleates through the layer-by-layer slip of partial dislocations on consecutive close-packed atomic
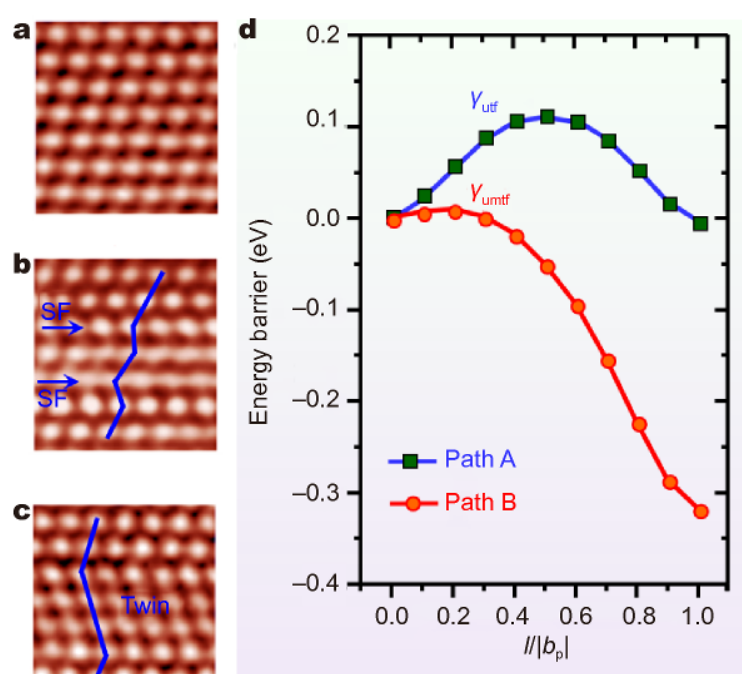

Figure $1(\mathrm{a}-\mathrm{c})$ In situ Cs-corrected HRTEM images showing the nucleation process of a deformation twin. (d) Density function theory calculation results presenting the energy barriers of deformation twinning. The calculated energy change as a function of the sliding displacement $l$ (normalized by the magnitude of twin partial slip $b_{\mathrm{p}}$ ) at the $\mathrm{SF}$ layer for paths $\mathrm{A}$ and $\mathrm{B}$. The energies of the initial states in $\mathrm{a}$ and $\mathrm{b}$ are set to zero.

planes and that deformation twins should be suppressed in many face-centered cubic (FCC) metals with high unstable twin-fault energy, such as $\mathrm{Al}$, $\mathrm{Pt}$ and $\mathrm{Pd}$, in which the formation of twin embryos is difficult [6]. However, this is at odds with extensive experimental observations of deformation twins in these FCC nc metals [7-9]. Recently, Han and his colleagues presented atomicresolution in situ evidence of a new route of deformation twinning in nc Pt having a high twin-fault energy [5]. An atomic-scale twinning process was captured in situ in a Cs-corrected transmission electron microscope (TEM). Fig. 1a-c shows a series of high-resolution TEM (HRTEM) images of a twin nucleation process, viewed along a $<110>$ direction and acquired $0.5 \mathrm{~s}$ apart during

School of Materials Science and Engineering, North Carolina State University, Raleigh, NC 27695-7919, USA

*Email: (ytzhu@ncsu.edu) 
an in situ tensile test. Fig. 1a shows an HRTEM image taken before plastic deformation and hence no dislocation was observed. Further loading led to the formation of two stacking faults (SFs) separated by a single atomic layer, which are referred to as 1-3 SFs on the basis of the relative positions of the $1^{\text {st }}$ and $3^{\text {rd }}$ atomic layers involved (Fig. 1b). The two SFs resulted from the emission of two partial dislocations from a GB. As shown in Fig. 1c, with further straining, one more SF formed in between 1-3 SFs, leading to the nucleation of a three-layer twin. This process of twin nucleation is intrinsically different from the previous twinning modes in which a twin embryo forms through the successive emission of partial dislocations layer-by-layer on consecutive $\{111\}$ planes [69]. It also offers a possible explanation on the nucleation of a type of twins without macroscopic strain with the cooperative slip of three partials with the sum of their Burgers vectors equaling zero [10].

$A b$ initio density function theory (DFT) calculations (Fig. 1d) indicate that the energy barrier for the classical layer-by-layer twinning route (path $\mathrm{A}$, denoted as $\gamma_{\mathrm{utf}}$ ) is much higher than the energy barrier of the new twining route (path $\mathrm{B}$, denoted as $\gamma_{\mathrm{umtf}}$ ). The lower twinning energy barrier for path $\mathrm{B}$ allows the bypassing of the high twin-fault energy barrier in $\mathrm{nc} \mathrm{Pt}$, thereby significantly increasing the possibility of twinning in nc Pt. This explains why deformation twins were frequently observed in experiments, even highly unfavorable in those metals with high unstable twin-fault energy such as $\mathrm{Al}, \mathrm{Ni}, \mathrm{Pt}$ and $\mathrm{Pd}$.

This new insight into the twinning mechanism benefits from the application of the in situ atomic resolution mechanical testing technique [3-5]. The atomic-scale dynamic processes demonstrated that the abundant steps at the grain boundaries facilitate the nucleation of separated but closely spaced partial dislocations that enables effective bypassing of the high twin-fault energy barrier for twinning. Their findings together with the grain boundary plasticity activities $[3,4]$ shed light on the key roles of atomic structures of grain boundaries in the plasticity of nc metals, which will help with the design of ultra-high strength and ductile metals.

Received 6 January 2018; accepted 7 January 2018;

published online 11 January 2018

1 Meyers MA, Mishra A, Benson DJ. Mechanical properties of nanocrystalline materials. Prog Mater Sci, 2006, 51: 427-556

2 Schiøtz J, Jacobsen KW. A maximum in the strength of nanocrystalline copper. Science, 2003, 301: 1357-1359

3 Wang L, Teng J, Liu P, et al. Grain rotation mediated by grain boundary dislocations in nanocrystalline platinum. Nat Commun, 2014, 5: 4402

4 Wang L, Han X, Liu P, et al. In Situ observation of dislocation behavior in nanometer grains. Phys Rev Lett, 2010, 105: 135501

5 Wang L, Guan P, Teng J, et al. New twinning route in face-centered cubic nanocrystalline metals. Nat Commun, 2017, 8: 2142

6 Van Swygenhoven H, Derlet PM, Frøseth AG. Stacking fault energies and slip in nanocrystalline metals. Nat Mater, 2004, 3: 399403

7 Zhu YT, Liao XZ, Wu XL. Deformation twinning in nanocrystalline materials. Prog Mater Sci, 2012, 57: 1-62

8 Chen M, Ma E, Hemker KJ, et al. Deformation twinning in nanocrystalline aluminum. Science, 2003, 300: 1275-1277

9 Liao XZ, Zhou F, Lavernia EJ, et al. Deformation twins in nanocrystalline Al. Appl Phys Lett, 2003, 83: 5062-5064

10 Wu F, Zhu YT, Narayan J. Macroscopic twinning strain in nanocrystalline Cu. Mater Res Lett, 2014, 2: 63-69 